VOL. 16 (1977), 306.

\title{
The automorphism group of a scattered set can be non-commutative
}

\section{Isidore Fleischer}

Perhaps the simplest example is the lexicographic sum of copies $z_{n}$ of the integers indexed by the integers. If one performs the shift in the 0 -indexed summand $m_{0} \rightarrow(m+1)_{0}$ while leaving all the other summands fixed, and follows this with the shift $z_{n} \rightarrow z_{n+1}$ in the index set, then the element $0_{0}$ will be sent on $1_{1}$; whereas under these automorphisms performed in the other order, it will be sent on $0_{1}$. This noncommutativity contradicts Theorem 9 in [1].

\section{Reference}

[1] J.L. Hickman, "Groups of automorphisms of linearly ordered sets", Bul2. Austral. Math. Soc. 15 (1976), 13-32.

Centre de recherches mathématiques;

Université de Montréal, Montréal,

Canada.

Received 4 January 1977. 